OF THB

CAUSES, DIAGNOSIS, AND TREATMENT

OF

\section{OBSTRUCTIONS OF THE INTESTINES.}

BY JOH N BURNE, M.D.,

Whow of the Royal College of Physicians, late Physician to the Westminster Hospital, Lecturer on the Practice of Medicine, \&c., \&c., \&c.

Read at the Quarterly Meeting of the Bath and Bristol Branch, March 26.

The frequency of obstructions of the intestinal canal, the variety of causes, the difficulty of diagnosis, the diversity of opinion as to treatment, and the imperfect consideration given to the subject hitherto, have withuut doubt determined the Royal College of Surgeons to select for competition "The Causes, Diagnosis, and Treatment of Obstructions of the Intestines within the Abdomen."

The danger that always attends and the fatal issue that too often results from intestinal obstruction have induced me to bring this subject before the Association, satisfied that the members recognise its great practical importance, as well as the great principle that the alleviation and cure of disease is the proper aim of all our studies, researches, and labours; satisfied, also, that the collective experience of this assembly will go far to determine the most rational and most promising treatment.

The causes of obstruction may be divided into-

1st. Intrinsic, or those causes which affect the intestine from within.

2nd. Extrinsic, or those causes which affect the intestine from without.

3rd. Those causes which do not range themselves under either of the foregoing divisions.

The intrinsic causes embrace-

a. Accumulations of ingesta, taken as food or otherwise.

b. Pathological, or, more correctly speaking, pathanatomical conditions of the intestines, namely, intra-intestinal tumours, stricture from scirrhus or other organic cause, and organised bands stretching across the channel of the bowel.

c. Enteroliths, or intestinal concretions.

The accumulation of ingesta, in the form of residuary alimentary matter or freces, may be seated in the small intestine, in the cacum, the colon, or the rectum; in the cæcum more commonly as an impacted mass, in the colon as a mere accumulation, in the rectum as a tough or friable hard ball: the last more generally in females. In addition to these causes are ingesta of an insoluble indigestible character, as pieces of bone, of sinew, of apple, fruit stones, seeds, magnesia, sulphur, and the like; also bard substances accidentally or intentionally swallowed, of which medical records furnish a catalogue of extraordinary instances.

Of the pathological causes. stricture from scirrhus is the most frequent, and is located more generally at the termination of the sigmoid colon in the rectum, often indeed in the rectum itself, but here, though it narrows, it rarely obstructs the channel of the bowel completely.

In the cacum, too, disease is not uncommon, the morbid condition being contraction of its cavity from thickening and induration of the submucous and subserous areolar tissue, the product of previous inflam. mation; but this contraction, again, seldom leads to complete obstruction.

The most remarkable cause of obstruction from morbid growth is that of organised bands stretching across the cavity of the gut, like a net-work, entangling the fæces, and producing sooner or later a total obstruction. These bands are met with in the rectum likewise, and are supposed to result from a muco-enteritis with effusion of fibrin and consequent adhesions, which, acted on by the peristaltic power of the bowel and the fæculent matter, become elongated, and persist thereafter in the shape of organised bands. Obstruction in the rectum from this cause may be only partial, in the cæcum complete, of which a remarkable case is related in my first "Memoir on the Cæcum and Appendix," in the 20th Vol. of the "Medico-Chirurgical Tiansactions."

Enteroliths are formed either in the intestinal canal itself, and are true intestinal concretions, or they are formed in contiguous viscera, and find their way into the intestinal canal; as, gall-stones and pancreatic calculi. Those originating in the intestinal canal may form upon a nucleus of effinsed fibrin or blood; or around some foreign body by the aggregation of the salts of lime and other matters; or may be formed of indigestible fragments of food, as woody fibre, the husks of fruit, and the like. So in countries where oatmeal is a common article of diet-in Scotland for instance, they are made up of the husks and beards of oats.

According to the authority of the Monros, many concretions have been found in the same individual. A single concretion may lodge and produce a complete and fatal obstruction; or may become impacted in a diverticulum. or in the appendix vermiformis cæci, where, by continued irritation, it may produce a perforative ulceration, followed by peritonitis and obstruction, in this case sympathetic.

The extrinsic causes embrace-

Strangulated hernia.

Adhesion of a convolution of intestine, aiter the operation for strangulated hernia.

Twist of the sigmoid flexure of the colon.

Diverticula.

Pseudo-membranous bands attached to the mesentery and peritoneum.

A pouch or hole in the mesentery.

Tumours extra-enteric.

The adhesion of the opposite free surfaces of a convolution of intestine, previously strangulated and relieved by operation, has been witnessed by myself in one instance. It is rare, because of the precaution taken by surgeons after the stricture has beeu divided to draw out the gut, and to separate any adhesions before returning it into the abdomen In the instance meutioned, the operation had been performed by an 
excellent surgeon, the late Mr. White, and it is probable the agglutination may have again taken place after the return of the gut. It is worthy of remark, moreover, how slight an ugglutination, separable by the least force, may be sufficient to obstruct the action of the bowel and prevent recovery.

Twist of the sigmoid colon, with or without laceration, is favoured by a relaxed state of the meso-colon, and may be caused by a sudden blow or movement; or, as O'Beirne suggests, by the sudden propulsion into it of excrementitious matter from above. The twist may be half a rotation, or, it may be a turn and a half, sufficient in either case to produce an invincible obstruction.

Twist of the small intestine, the axis being the mesentery, occurs also as a cause.

The diverticulum is an abornal appendix to the small intestine, often scveral inches in length and wide as the bowel itself, communicating openly with the gut, but closed at its distal extremity. When congenital, it often hangs unattached in the abdomen, but occasionally a band proceeds from its blind extremity and adheres to some point of the mesentery or peritoneum, and under this band convolutions of intestine may become strangulated and cause obstruction.

Pseudo-membranous formations in the shape of organised bands, the product of previous inflammation, may also incarcerate the intestine. To these the female sex, in the opinion of Rokitansky, is more prone than the male, the pseudo-membranes being frequently attached to the internal sexual organs.

The third division of causes includes-

Intus-susception.

Enteritis.

Colica a plumbo, and

Spasm of the intestine.

A spasmodic con tric ion, though rare, must be reconnised as a cause of obstruction. In a case which occurred to Dr. Todd, and proved fatal in about thirty bours, there was found a contraction in the lower porticn of the ileon, from which the transition to the dilated pcrtion above was abrupt, and there was no mark of external compression of any kind.*

Of 169 cases of obstruction, collected with great industry by Mr. Benjamin Phillips-

63 were instances of invagination.

16 were tumours pressing from without.

19 were the results of stricture from diseaso of the parietes.

11 were the result of intra-intestinal tumours, hardened fæces, or concretions; and

60 were caused either by constriction, by bands, by adhesion, by the passage of the intestint through some abnormal opening, or by a twisting of the intestine upon itself.

Of the 169 cases, 133 tertminated fatally, viz. 7 out of 9 , or about 78.7 per cent.

Seeing that the causes of obstruction are so many and various, the diagnosis becomes a question for our

* Observations on Inteblinal Obstructions, ...c, by Benjaw.n Pbilijs, R.S., Med. $\mathrm{C}$ I i. Tians., Vol. xxxiij., p. 6. earnest consi leration, as upon it the principle of treatment much depends. In the exploration of the abdomen, by touch and by percussion, too great pains cannot be bestowed; the attention, at the same time, being alive to the fact that disease is prone to establish itself at those parts of the intestinal canal where the dimensions vary and the organization changes.

Holding in mind all the causes of obstruction, we may best guard ourselves against overlooking any; and by comparing the symptoms present in any particular case with those which are known to be proper to some and common to several, we shall be able to cast out from the list one after the other till we arrive at the single true cause, or reduce the number to a narrow limit of remaining causes, which have much in common, and to which the same treatment is applicable. By pursuing this plan of analysis the risk and fatal error of overlooking a strangulated hernia may assuredly be avoided.

In our inquiry into the special symptoms of each cause, or group of causes, we should be exact in our examination of the situations of hernia, not forgetting that strangulation may exist at the inner ring of the inguinal canal, and offer but slight evidence of tumour, especially in a stout person, Or, tumour being present in a hernial site, doubt may exist as to whether there is or is not strangulation, and an exploratory operation be required. A case of this nature occurred to myself, in which, in consultation with Mr. James, of Exeter, it was deemed advisable to explore by incision an elastic swelling at the navel.

Intus-susception, internal strangulation, and twist of the sigmoid colon, rank in the same category and have signs in coinmon; as sudden attack and great suffering, perhaps after some strain or exertion, the patient having up to the moment of seizure been in his usual health. Signs referred to the left ilio-inguinal region may point to the sigmoid colon, while deep-seated circumscribed tenderness, with resistance to the touch and dulness on percussion, may point to strangu'ation or invagination, to the latter especially if blood is voided from the intestine, a sign in this case almost pathognomonic. Yet doubt will exist.

Impaction of faces in the cacum may be recognised by a distinct circumscribed tumour in the right ilioinguinal region, in conjunction with a costive habit; accumulation in the colon by a solid feel in the course of the git, with dulness on percussion; impaction in the rectum by urgent tenesmus, verified by digital exploration.

Scirrhous disease at the termination of the colon in the rectum, may form and exist without any other symptoms than those of dyspepsia, attended often with a diptheritic or apthous state of the mouth-a suspicious sign; the action of the bowels, formerly regular, having become difficult and uncertain, the dejections at the same time being scanty, soft, and very offensive; followed, sooner or later, by complete obstruction. Pains of a neuralyic character in the abdomen and about the I runk of the body are the frequent attendants of organic disease of the intestine, tending to occlusion of the canal. 
The symptoms described may excite suspicion, and suggest the necessity of examining the gut itself, in doing which tact is required, for if the course of the rectum be followed the finger will be lost in the hollow of the sacrum. To reach the colic extremity of the rectum the index finger should be introduced up to the knuckle, and directed across the pelvis from the coccyx to the projection of the sacrum. Not holding in mind these particulars, two persons failed to detect a scirrhus in ti.e case of a gentleman who had been under my care, and in whom the symptoms led me to suspect disease, which, on examination, I was just able to detect with the tip of the fi:Iger at the colic extremity of the rectum. On this being announced a consultation was desired, and another physician was called in. He failed to reach the disease; upon which a surgeon was requested to meet us. It happened that the surgeon, not being able to keep bis appointment, risited the patient alone, and, having made an examination, assured him that there was no disease. Next day we all met, when both these gentlemen were able to satisfy themselves of the existence of a scirrhous stricture.

The existence of spasm as a cause is indicated by intense exacerbating pain, restlessness, the absence of febrile movement and of the other symptoms of inflammation, and by the successive development of the signs of obstruction.*.

The signs common to all obstructions are constipation, pain, vomiting, and depression of the powers of life; and in direct proportion as these are sudden and violent so is the danger. So tight sometimes is the strangulation from a diverticulum that gangrene and death will ensue in less than forty hours. $†$ The violence of the symptoms may in some degree assist our diagnosis. In the obstructions from facal accumulation the countenance does not betray extreme suffering, nor

- Ileus.-Spasmodic contraction of the lleon; Death in about sixty hours.

- - aged 5 years, was admitted into the clinical ward, loydia, No.7, Guy's Hospital, on Sundav, the 2 , th of November, 18 11, pt 11 o'clock, A.M., under the care of Dr.Cholmelev. On admission, the hoy complained of scvere exacerbating pain in the abdomen, and cri d much. The rbdomen on exploration presented nothin' abuormal. The litule patient was restless, moving about the bed from si !e to side, generully on his hands and knees, sometimes lying on his back. When on the hack he drew his limbs up; when on his knees he would move his hrad from side to side in agony. The tongue was yellow sh and thickly furred, the face ser pale, the features contracted, and marked expr.ssion of great suffering; pulse 156, rather sharp ; constipatinn.

It was stated that on the previous evening, Saturday, the boy was gnize', while spinning bis top, with a violent pain in the belly, which had continued until his admission. He had taken rhubarb and was nuw ordered castor il and a fomen ation.

In the evening of Sunday, vomiting supervened, the belly was becoming tense, and his sufferings aggravuted. Hirud. xii. abdomini; ralomel gr.ss ; Opii, gr. $\frac{1}{3}$; every three hours. At ten P.M., enesection to tive ounces, followed by temporary relief, but the pain havin $x$ sunn resumed its violence, $\mathrm{Hi}$ ud. xii. were repeated. These 1 ech bites bled all night, and al though on Monday the pain abated, no dej ction oc urred, and the little fel'ow oeing very much exhatsted died on Tuesday morning earl, about sixty hours fiom the ttack. A tew minutes atter he appeared to be dead, a great quantity of treces passed.

On dissection eighteen hours atter $d$ ath, the onlv abnormal condition discovered was contraction of the small intestine in threc places : one several inches in length, the two others annular and the inches apat inclosing in the intervening spact faculent matter, heterogeneuus, many coloured, and undigested, like the $g$ ip ng stool of a child.

This nust be regarded as an example of obstruction from spasm of the sma'l intestine, produced by the irritation of its contents; and death may have resulte.l partly from the intense suffering, partly from exhaustion following the excessive loss if bl.od.

† Medical Times, August 24tb, 1850. II. W., ag: 19, in 39 hours. is the general aspect that of imminent danger; accordingly, these cases will hold on day after day, yielding at length on the fourth or sixth, or as late as the tenth day.

A sign of some interest, and in part diagnostic, is the powerful peristaltic action often observed, so powerful as to be obvious to the touch and sight, like a snake coiling and moving in the abdomen. This effort of nature to overcome the obstacle is a sign common to most obstructions where the cause is mechanical; with the exception, however, of strangulation, in which, as in enteritis, there is a perfect slillness in the abdomen. This differential sign, if verified by others, would deterinine between obstrnction from strangulation and from other rnechanical causes.

Tenesmus and resistance to the passage of enemata point to the rectum or sigmoid colon as the seat.

Blood roided per alvum indicates invagination.

Tumour, deep-seated resistance to the touch, with dulness on percussion and pain and teriderness, indicate the point of obstruction; also the point is indicated when injections reach a certain spot and there stop, and the intestines propel their contents downward to the same spot and no further. It is said that the vomiting and pain are more severe when the obstruction occurs in the small than in the large intestines, and there may be some truth in the remark, but the exceptions to the rule are many. It has also been said that if the urinary secretions be copious the obstacle must be far removed from the stomach, and vice versa ; but the exceptions to this rule are also many.*

With every aid that our present knowledge can supply, the diagnosis will often be perplexed in consequence of the great diversity in the situation of the c lon, and of other abnormities in the cavity of the abdomen.

The treatment of obstruction of the intestines will depend much on the opinion formed of its cause. Should a strangulated hernia be discovered, the established remedies leave no doubt as to the course to be pursucd. Should the symptoms favour the belief that the obstruction is caused by an accumulation of fæces, the question at once arises,-What, and to what extent, purgative medicines should be administered? a question to be determined partly by the acute character of the symptoms and partly by the manner in which purgatives are borne by the stomach.

In any case it may be proper, at the outset, to give purgatives in strong doses, as colccynth, calomel and opium, followed by senna and saits, the dose to be repeated in six hours. But these proving ineffectual, are we to persist in the further and frequent use of them ?

The presence of a fæcal obstruction seems so naturally to call for the aid of purgatives, that one is tempted almost irresistibly to perse ere in their administration, even though the stomach reject them; and such has been the too general piactice. But observaion and experience teach us to pause in this course, so frequently do we find that the strongest purgatives, resolutely

- Phillips' paper, p. 17 . 
administered, are not only given in vain, hut have a prejudicial effect, which compels us to desist; and yet by and bye the bowels act and the patient recovers. To what extent then are we called upon to prescribe purgatives? My own experience decides in favour of limiting their use, and the experience of the profession is fast tending in that direction.

It must be remembered, that often the intestine above the obstruction is itself making most powerful efforts to overcome the obstacle, as is evidenced by the striving action of the convolutions attended with acute suffering. Can good, then, arise from urging the intestine to greater efforts? We may truly answer-No. On the contrary, serious harm; for the irritation of purgatives may aggravate the tendency to inflammation, a tendency always present; and certain it is, that they aggravate the irritability of the stomach, encourage and increase the vomiting, and combine with the disease to exhaust the powers of the patient. This they do by exciting not merely more frequent vomiting, but by actually inducing a secretion from the stomach and upper portion of the intestinal canal to an extent which drains the blood of its more fluid constituent, exactly as does the Asiatic cholera. The continued use of purgatives, then, is objectionable on this score, besides that it is ineffectual.

On what remedies then are we to rely? Calomel may be adnissible once in twelve hours, in full dose, if the stomach do not reject it; but the remedy that gains favour by experience, and promises the best results, is opium, crude in the first instance, afterwards in the form of the salts of morphia.

The criterion of the extent to which opium should be given is the degree and frequency of the pain, and on this we may fairly rely. Opium in the dose, first of a quarter then of half a grain, and later the acetate of morphia in the dose of a quarter of a grain, may be repeated every four hours so as effectually to relieve the pain; and, if it should narcotize the patient in any slight degree, so much the better.

A very instructive example of the propriety of this treatment occurred at Tiverton, in January, 1850, my friends Mr. Gervis and Dr. Paterson, in cunjunction with myself, being in attendance. In this case purgatives were given with perseverarce till their ill effect in keeping up the vomiting and aggravating the throes of pain was so obvious, and the powers of life were sinking so rapidly, that we were of one mind as to the necessity of suspending them and of relying on opium. This course having been adopted, the romiting diminished, the morphia soothed the pain, the patient slept during the night, and the obstruction yielded on the following day.

In another case to which I was called in consultation, some years ago, every resource had been tried, fæculent romiting was present, the powers of life were at a low ebb, and all treatment was abandoned, morphia excepted, which, in doses of a quarter of a grain, was exhibited as the throes of pain returned. On the tenth day the obstruction yielded and the patient recovered. Very lately also, a case of obstruction has been treated at
Guy's Hospital successfully with opium, to the exclusion of other means.*

Although experience may decide us to abandon the frequent repetition of purgatives, it sanctions the occasional exhibition of a saline aperient, -as the soda potassio-tartras, in the state of effervescence, which salt, in the dose of one drachm, often proves grateful, and tends to liquefy the fæces; but even this should not he repeated oftener than once in twelve hours, as, independent of other reasons, salts produce disfressing thirst.

In obstruction from fæces impacted in the cæcum, there being seldom so much irritability of the stomach, purgatives are more admissible, and, conjoined with calomel and opium, constitute the main treatment. Here, however, saline aperients are particularly valuable; and it does happen, that the stronger purgatives of senna, salts, and jalap, are efficacious. Yet, as the impacted mass requires time to be liquefied, purgatives should not be pressed too assiduously. When fæces are impacted in the rectum, the mass requires to be broken up, and extracted by mechanical means. When the symptoms lead to the inference that the obstruction is either from internal strangulation, twist of the bowel, or intus-susception, we recognize here invincible obstacles which forbid the use of purgatives in any form or dose. All the resources which medicine can supply avail nothing. Under these desperate circumstances, with no other prospect than prolonged torture, and inevitable death, desperate remedies are justified, may we not say demanded? The obstruction admits of relief if the parts involved could be got at, and surgery has made the bold attempt. My friend Mr. Hilton has opened the abdomen twice, though unsuccessfully, and has had occasion to regret the omission of the operation several times, once in the last summer, where a postmortem examination proved the diagnosis to have been correct.

However hazardous to life wounds of the peritoneum may be, the dread which formerly deterred surgeons trom making incisions into the abdomen no longer exists; they are of constant occurrence in operations for hernia, and recovery after them is common. Dr. F. Bird has made small incisions into the abdomen in eighteen cases as a means of diagnosis or relief, and in no case did a bad result ensue from such incisions. Nor is recovery unfrequent after the incisions of great extent in the modern operation of ovariotomy. On this ground, then, need we hesitate? That which makes men unwilling to risk an operation, is the doubt which involves every case as to the exact seat and nature of the obstruction. But doubt will ever remain. Weighing all the circumstances, and judging as best we may of the seat of the obstruction, and an operation having been determined on, is it advisable to open the abdomen at the particular spot? In cases where the nature of the obstruction is clearly indicated, the incision may be made as near as practicable to that spot; but where the point of obstruction is not well defined, if the abdomen 
is opened on one side, and the cause of the obstruction proves to be on the other, the operation will have been performed in vain; and the probability of such a result is great. Only within a few weeks two friends of mine differed in opinion, the one thinking the obstruction was near the cæcum, the other in the sigmoid colon. The same difference of opinion existed between Recamier and Dupuytren, two eminent men.* How then decide? In the midst of such difficulties would not the large incision in the median line, as practised in the Cæsarian section and in ovariotomy, be preferable? Would it not afford the best chance of discovering and removing the cause of obstruction, wherever seated ?

This proceeding my own opinion would countenance; it has been practised on various occasions by eminent surgeons-by Messrs. Hilton and Erichsen recently, and is recommended by Mr. Phillips, but its propricty must be decided by experience. $\dagger$

Of the treatment of obstructions from scirrhus of the rectum much need not be said. It is in vain to expect relief from medicine. Nor can relief be hoped for from attempts directed to the stricture itself, which force might lacerate, but could not dilate, and surgeons wisely desist. It may, perbaps, be possible to pass a gum elastic catheter through the stricture, even when high up, though I have seen the late Sir Astley Cooper make the attempt and fail ; but, supposing this accomplished, it wouid be hazardous to inject fluid with a view to liquefy the fæces and favour their escape, for force must be employed which would endanger the rupture of the colon, distended already to the utmost. One resource remains, scarcely preferable to death perhaps, but which it is our duty to suggest-the opening of the colon, after the plan proposed by Callisen and practised by Amusat and others. This may succeed, and an artificial anus being established in the left loin, life may be prolonged.

\section{- Phillips, ibid.}

† Extract from Mr. Phillips' paper.

Hevin, Menschidg, Boyer, Dupuytren discourage an operation. Bonnet agitates the question but leaves it to be resolved by experience. Manoury recommends gastrotomg before the inflammation is conviderable, the incision to be made near the spot indicated.

Otto says, when the powers of life are in all their integrity no reasonable surgeon would operate; but after all remedies have been einployed fruitlessly, the powers are so exhausted that the patient cannot bear up against the operation; or the bowel may have become gangrenous.

A case detailed by Velse in which Neck was consulted, pronounced the case intus-susception, recommended an operation, which was performed at the spot indicated. An invagination was found, the gut was gently withdrawn, and the patient survived twenty years.

Fuschius described a case of gastrotomy for invagination-successful. Phillips' Ibid.

Erichsen's case was twist of the sigmnid colon, the operation in the median line, the twist detected and relieved, Hatus and frces passed, the obstruction done away, but the patient sunk and died. The operation performed at nine A.M, lasted one hour, patient much exhausted. At five P.M. passed a fluid motion with much flatus, relief, spoke cheerfully. No return of vomiting after the operati $n$. At eight p.M. he passed zother fluid, faculent, and copious motion, but almost immediately afterwards sunk back on the pillow and expired, being eleven hours after the operation.

By examination post-mortem.-The obstruction was found to have existed at the end of the transverse colon in the left lumbar region, the meso-colon of the sigmoid flexure being loose and admitted of partial twist. The bowels empty, channel free, showing that the cause of obstruction had been removed by the operation.

Query.-Did not the patient die from exhaustion? He took beef-tea, but no mention is made of other support.-Braithwaite's Retrospect, vol. xxi, 18j0, p. 240, from Lancet, Jan. 25, 18j0, p. 108.
The feasibility of this operation is placed beyond doubt by no fewer than three 'successful cases, lately published in the 33rd volume of the Medico-Chirurgical Transactions; the operations having been performed respectively by $\mathrm{Mr}$. Field, Mr. Clarkson, and $\mathbf{M r}$. Pennell. The region, the left lumbar, selected for this operation, is most favourable, there being a space on the outer margin of the quadratus lumborum muscle, where the wall of the abdomen is thin, and admits of the colon being opened without wounding the peritoneum. By Mr. Field and Mr. Clarkson, the operation by transverse incision was preferred; by Mr. Pennel, that by the vertical incision. By Mr. Field, difficulty was exprienced in distinguishing the gut, fascia having been mistaken for it, a difficulty to be obviated by'recollecting that the tissues to be divided, as stated by Velpeau are-

The very thick skin.

The cellulo-adipose tissue.

The origin of the transversalis muscle or its aponeurosis, and a second layer of cellulo-adipose tissue; of which a mass lying between the colon and the transversalis must be dissected through, and much of the fat removed before the bowel can be reached.

The relief consequent upon this operation is complete. But, as time advances, a decided disposition in the outer orifice to contract manifests itself, and leads, eventually, to renewed difficulty and danger. Would tents of sponge densely compressed, as used by Dr. Simpson to dilate the uterus, have power, by expansion, to keep the orifice patent?

The inflammation developed in cases of obstruction may call for the abstraction of blood, either from a vein or by leeches; but, inasmuch as the inflammation is the consequense, not the cause, of the obstruction, blood should be drawn cautiously, with a view to its control, for it cannot be extinguished, the cause remaining. Moreover, when the cause of obstruction is not insuperable the signs of tenderness and pain (which would seem to demand the loss of blood) are due to irritution and spasm rather than to inflammation, and are best relieved by opium; and hence the value of this remedy. The alleviation of pain, indeed, is a main point in the treatment of obstruction from any cause, for pain may destroy life; and in proportion as pain is urgent so should opium be given. In a case related to me by Sir Astley Cooper, of obstruction caused by the lodgement of a concretion in the ileon, the excruciating pain destroyed life in eight hours.

If blood is drawn too freely at the outset it would leave the patient ill prepared to bear up against prolonged suffering with want of nourishment, and might in this way turn the balance against him. In the case of intus-susception, not relieved by operation, the only chance of life is the separation of the invaginated portion of the gut, gangrene having first occurred; a process which requires time: so that if the powers of life have been reduced by excessive blood-letting, as well as by the disease, the patient will sink before nature can accomplish her task. Blood, therefore, should be drawn with circumspection. 
Fomentations and warm baths are valuable adjuvants; they soothe pain, relax spasm, and, by diminishing suffering, save power.

Of all the remedies at our command, enemas in conjunction with opium, are perhaps the most essential, and, where the obstruction is not invincible, contribute more than any others to bring about a happy termination. Enemas, composed of bland fluids, should be injected twice in the day, to the fullest extent the bowel will receive, by the aid of O'Beirne's colon tube, a most valuable instrument in these cases.

Among the other remedies employed as a last resource are tobacco, fluid mercury, the cold douche, and galvanism; the two last said to be successful occasionally. But mercury is of no use as far as I have seen, and is otherwise open to great objection. Tobacco is a valuable, because often a successful, remedy; but on account of its poisonous properties it is administered only in the form of enema, the infusion for which, on the score of safety, should not be stronger than fifteen grains to a few ounces of boiling water.

Strychnia may deserve notice, and has been given in one case, in the dose of 1-16th of a grain dissolved in distilled vinegar, with remarkable success.

I have now brought to a close a subject of deep interest, treated with prolixity I fear, but not exhausted. Saldom is the mellical practitioner placed in a more distressing situation than when attending a case of obstrucition he fiads his resources exhausted, but the obstacle not overcome. Surrounded by anxious relatives, tormented with suggestions by obtrusive friends, he has to watch, through many long hours, suffurings which he cannot relieve, and at the last to witness a death he cannot avert. Fortunate shall I esteem myself if the consideration given to this subject, and the practical opinions elicited from this assembly, should fortify our resources, and qualify us to treat with better success these formidable obstructions of the intestinal canal.

Bath, March 26, 1851.

\section{CASES OF}

\section{AFFECTIONS OF THE NERVES} Dergisuted To ras

\section{EYE AND ITS APPENDAGES.}

\section{By AUGUSTIN PRICHARD, EsQ.}

\section{Read at the Quarterly Meeting of the Bath and Bristol Branch, March, 1851.}

Affections of the Crantal Nerves, especially those distributed to the Eye and its Ap. PENDAGES.

The following cases of paralysis and spasmodic affections of the nerves of the orbit are selected from many which have fallen under my notice, not so much for the purpose of showing the results of treatment when it was adopted, as to illustrate the functions of the particular nerves. I take the nerves in their numerical order, as they are known to all, and with one or two exceptions have omitted a detail of the cases which are most commonly noticed.

Loss of function in the optic nerve, (or 2nd pair,) is unfor.unately sufficiently common, producing amaurosis in its various degrees; I have therefore included in the present report none of the ordinary instances of this disease. The following case was one of complete blindness from amaurosis occurring many years ago, having been under my father's care in the Infirmary here, and I extract this report from my notes made at the time.*

Case 1.-A young man, a painter by trade, and 13 years of age, was admitted in a state of total blindness. He was seized with a fit a few days before, and lost his sight from that time. He was accustomed to work a gri at deal in oil and lead paints, and at the time of his seizure was suffering from the effects of mercury, administered for the cure of syphilis. He remained in the wards of the infirmary for nearly nine months, and was then dismissed with his sight completely restored. During the whole of his stay he was treated upon one plan, and with the greatest activity and perseverance. The sight began to improve after he had been about a month under treatment. The following abstract of the treatment will give an idea of the extent to which it was considered necessary to push the antiphlogistic plan, and the result obviously justified it:- He had, at intervals, no less than twenty dozen leeches applied to the head; he was bled from the arm five times, and on one occasion Itill syncope occurred; he was bled once from the temporal artery ; he had blister: $t$, his scalp; a long issue was made about a week after his admission, and after a time a seton was made in the back of his neck; he was, moreover, during the greater part of the time, taking active purgative doses, and for a short time he took the decuction of sarsaparilla with the iodide of potassium. His mouth was also affected with mercury soon after he was admitted.

In drawing attention to this as one of the very few cases of complete amaurosis that are cured, I must express my belief and fear that the patient, if he was treated now, would have so much less chance of recovery, as the treatment would be, in all probability, less active and decided.

Case 2.-Sarah Merchant, aged 21, admitted June 20th, 1849.† Has had an affection of the right eye for two years, which gives rise to considerable inconvenience. She sees nothing with the outer balf of the right eye,-that is to say, when the left eye is closed, she sees only that half of an object directly before her which is towards her right hand.

This was a well marked instance of partial amaurosis,

- I have found, since $I t$, anscribed this case from my notes, that it was alluded to by my father in a paper "On Cerebral Diseases and their Truatm " + This and the following cases were admitted at the " Bristol Dispensary for the cure of complaints in the eyea." 